\title{
Bienestar Social, Económico y Ambiental para las Presentes y Futuras Generaciones
}

\author{
Lilia Fernández y Mirella Gutiérrez \\ Universidad Autónoma Metropolitana unidad Azcapotzalco, División de Ciencias Básicas, Departamento \\ de Ciencias Básicas, Área de Química Aplicada, Av. San Pablo No. 180, Col. Reynosa Tamaulipas, \\ CP. 02200, D. F.-México (e-mail: Ifs@correo.azc.uam.mx)
}

Recibido Sep. 13, 2012; Aceptado Nov. 14, 2012; Versión final recibida Ene. 22, 2013

\begin{abstract}
Resumen
Este trabajo tiene como objetivo abordar la diferencia entre desarrollo sostenible y sustentable, aclarando cual describe el desarrollo humano social, económico y ambiental, respetando al ambiente. Es un trabajo de reflexión documentada que arroja en sus hallazgos el uso del término "sustainable" en la literatura Inglesa conforme al informe Brundtland. Su traducción al castellano como sostenible, la incorrecta traducción al castellano por sustentable y el concepto Latinoamericano de los términos desarrollo: sostenible y sustentable como dos vertientes diferentes del desarrollo, son analizadas. El desarrollo sustentable (insostenible) es un crecimiento económico que no atiende el deterioro ambiental y social que ocasiona. El desarrollo sostenible es el desarrollo con futuro que garantiza la satisfacción de las necesidades de las generaciones actuales y futuras sin comprometer los recursos naturales y humanos. Se concluye que el término desarrollo sostenible es "sustainable development", descrito por la Organización de Naciones Unidas para el desarrollo integral del hombre.
\end{abstract}

Palabras clave: desarrollo sostenible, desarrollo sustentable, deterioro ambiental, deterioro social, bienestar

\section{Social, Economic and Environmental Welfare for Present and Future Generations}

\begin{abstract}
The objective of this article is to discuss and clarify the difference in Spanish between development "sostenible" and "sustentable", explaining which describes human development in the social, economic and environmental areas. This is documented reflection that discusses and analyzes the use of the term "sustainable" in the English literature according to the Brundtland report. Its translation into Spanish as "sostenible", the incorrect translation into Spanish as "sustentable", the Latin American concept of the terms: "desarrollo sostenible" and "desarrollo sustentable" as two different development streams are analyzed. "Desarrollo sustentable" is the economic growth that does not consider the environmental and social damage that it causes. "Desarrollo sostenible" is the development with a future that ensures the fulfillment of the needs of present and future generations without compromising natural and human resources. It is concluded that the Spanish term "desarrollo sostenible" is "sustainable development", described by the United Nations Organization for the integral development of the human beings.
\end{abstract}

Keywords: sustainable development, supportable development, environmental damage, social damage, welfare 


\section{INTRODUCCIÓN}

El objetivo del trabajo es establecer de los términos desarrollo: sostenible o sustentable, el que se refiere al establecido en el informe Brundtland "satisfacer nuestras necesidades actuales sin comprometer la capacidad de las generaciones futuras para satisfacer las suyas" (ONU, 1987), que implica la protección del planeta y la permanencia en él del hombre de manera equitativa, viable y vivible es decir con bienestar social, económico y ambiental hoy y mañana. En el contexto de la conferencia de la Organización de Naciones Unidas ONU sobre medio ambiente y desarrollo, concebida como la Cumbre de la Tierra (1992), se estableció para el desarrollo sostenible la protección ambiental. Debido a la relación entre desarrollo económico, social y ambiental se da relevancia a la eliminación de la pobreza y la reducción de desigualdad de nivele de vida.

En la conferencia sobre medioambiente y desarrollo humano en la Cumbre de la Tierra Rio+20 celebrada del 20 al 22 de julio del 2012 en Rio de Janeiro, se reconoció el poco avance que en materia de sostenibilidad se ha logrado y en este trabajo se hace hincapié en el concepto del desarrollo sostenible y el deseado para el bienestar humano hoy y mañana, y para hacer conciencia entre los docentes de cambar su forma de pensar y sentir, para educar en la sostenibilidad (Educación para la sostenibilidad, 2005; Novo, 2009).ya que la educación es el medio más efectivo que la sociedad posee para enfrentar los retos del futuro. En efecto la educación le dará forma al mundo del mañana (UNESCO, 1987).

Naredo (1997) señala dos tipos de nociones de sostenibilidad que responden a diferentes paradigmas. Una sostenibilidad débil formulada desde la economía estándar (Castañeda, 1999) y una fuerte (Falconi, 2002) formulada desde la racionalidad de la economía física que es la termodinámica y de la economía de la naturaleza que es la ecología. Garmendia et al., (2010) conceptualiza a la sostenibilidad débil como la reducción de existencias y favorable desempeño económico, y no considerar los impactos irreversibles en el ámbito ecológico, socio-económico o cultural. La sostenibilidad fuerte, sostiene que muchos servicios fundamentales prestados por la naturaleza no pueden ser sustituidos en cualquier nivel por capital hecho por el hombre. La sostenibilidad fuerte, se preocupa por la salud de los ecosistemas en los que se inserta la vida y la economía de los hombres sin ignorar la incidencia que sobre los procesos del mundo físico tiene el razonamiento monetario. Es la sostenibilidad fuerte, la que puede responder a la sostenibilidad de ciudades y asentamientos humanos. Según Norton (1992) hay dos tipos de definiciones de sostenibilidad, la científico social como la del Informe Brundtland que relaciona el bienestar presente y futuro de las personas y las ecológicas, que requieren la protección de los procesos ecológicos como condición de sostenibilidad.

Sostenibilidad: Mejorar la calidad de vida humana viviendo dentro de la capacidad de carga o de sustentación de los ecosistemas de apoyo. Sostenibilidad ecológica: Para el ser humano la sostenibilidad es tener un medio ambiente vivible, una economía equitativa y procesos viables con una administración responsable del manejo de los recursos. Ecológicamente, la sostenibilidad se describe cómo la permanencia de los sistemas biológicos a seguir siendo diversos y productivos a través del tiempo, condición necesaria para el bienestar humano y de otros organismos. La sostenibilidad, no es una moda es una exigencia de los nuevos retos y reglas globales de competitividad que se están imponiendo en un entorno mundial de creciente demanda y escasez de los recursos naturales.

Sostenibilidad parcial o local: Es un subsistema que tiene todos los recursos para un largo plazo. Sostenibilidad global: Sistema que contiene a un subsistema sostenible razonado a largo plazo, si no es razonado, entonces se perderá la sostenibilidad del subsistema y por consiguiente del sistema global, llámese tierra, se volverá insostenible (Naredo, 1997).

Pearce et al., (1989) establece que en la sociedad sostenible no debe haber: declive no razonable de recursos, daño significativo a los sistemas naturales y declive significativo de la estabilidad social. Daly (1994), propone una sociedad sostenible como aquella en que: los recursos renovables no se deben utilizar a un ritmo superior al de su ritmo de regeneración, no se emiten contaminantes a un ritmo superior al que el sistema natural es capaz de absorber o neutralizar y que los recursos no renovables se deben utilizar a un ritmo más bajo del que el capital humano creador pueda reemplazar al capital natural perdido. Según la Comisión Económica para América Latina, CEPAL (2002), el desarrollo sostenible se apoya en el reconocimiento de la función que cumple el medio ambiente y los recursos naturales para garantizar el progreso económico.

Tres vertientes del desarrollo sostenible (Rodríguez y Govea, 2006): La primera se relaciona con el origen de la palabra; del latín Subvenir que significa mantener arriba o apoyar desde abajo, acorde a este sentido, una comunidad está apoyada en sus referentes históricos-geográficos, sus acervos culturales, sus habitantes actuales y futuros. De allí el sentido y el sentimiento de continuidad, de arraigo. La segunda vertiente alude a los intereses relevantes del hombre en su visión al futuro, de lo que desea construir y en 
qué valores va a apoyarse para levantar esa realidad societal y crear dispositivos consistentes con el logro de fines colectivos. Es importante el factor político relacionado con: la democracia, las formas de generación de consenso, el diálogo, estilos de negociación; y los mecanismos de construcción de ciudadanía y consolidación de capital social. La tercera vertiente implica la interrelación dinámica de la triada HombreSociedad-Medio Ambiente, proponiendo soluciones dirigidas al logro de la sostenibilidad en el sentido de reexaminar el rol de la economía; la concepción sobre la naturaleza; el papel de la ciencia y la tecnología, y especialmente los valores sobre los cuales se construye socialmente la conciencia ciudadana y su sentido de compromiso y responsabilidad social. Lo sostenible alude a considerar los siguientes parámetros: Los recursos y su utilización con criterios de adecuación y pertinencia. El desarrollo de alternativas que conduzcan a hacernos menos depredadores como especies. El empleo en la Industria de tecnologías limpias, más verdes menos contaminantes y más comprometidas con el bienestar humano. La consolidación de una cultura política orientada hacia el estímulo del compromiso y la responsabilidad social de los políticos y actores clave del proceso de gestión pública y empresarial. Revalorización de las actitudes y conductas ancladas en valores éticos y, sobre todo, que el Estado y las instituciones públicas creen dispositivos legales y de participación dirigidos, por una parte, a limitar el uso inadecuado de los recursos y por la otra, posibilitar la participación de la ciudadanía en la defensa de bienes sociales, culturales, económicos, naturales que constituyen el acervo sociohistórico y cultural de los humanos y de su medio ambiente

La esencia del desarrollo sostenible es satisfacer las necesidades humanas fundamentales al tiempo que se preservan los sistemas que soportan la vida del planeta, según la Asociación Americana Para el Desarrollo de la Ciencia (Kates et al., 2001). La realidad nos lleva a una disyuntiva entre satisfacer las necesidades incesantes del hombre y cómo preservar los recursos naturales. Un desarrollo de producción, consumo y enriquecimiento de las naciones también es de descuido por el medio natural y el equilibrio ecológico a nivel mundial y por tanto no es un verdadero desarrollo (Redclift, 1987). El sistema económico creado hace años y el modelo ambiental capitalista usado por los países desarrollados e industrializados podría ser la causa del deterioro de la tierra; los modelos de producción y consumo se basan en la explotación de los recursos naturales.

Sustentabilidad: Modelo productivo que no privilegia la protección del capital natural contra su explotación indiscriminada. Se le conoce también como desarrollo insostenible o de acuerdo a Reyes-Sánchez (2012) soportable (supportable en el idioma Inglés).

\section{DESARROLLO SUSTENTABLE O SOSTENIBLE (SOSTENIDO)}

Usamos el término desarrollo sostenible y sustentable como sinónimos de un desarrollo humano equitativo, viable y vivible pero realmente ¿es lo mismo? Probablemente el término sustentable sea un pochismo del término inglés sustainable. Desarrollo sustentable y sostenible tienen diferencia semántica, demográficas, culturales, valorativas y sobre todo de índole política, porque comprometen la existencia de vida del hombre y su entorno. En su trasfondo opacan las reales crisis ecológicas y ambientales producto de la actividad económica; la expansión del capitalismo hacia actividades emergentes cada vez más degradadoras del medio ambiente; la acción depredadora del hombre mismo cuando no se preocupa por su entorno, y las conductas utilitaristas carentes de sentido del nosotros (Leff, 2003).

¿Cuál término sostenible o sustentable? El Tecnológico de Monterrey, México resolvió el debate entre sostenible y sustentable a partir de lo que dictan los expertos lingüistas y señala en su foro Acuerdo por un México Sostenible (2011) que se utilizará el término sostenibilidad o sostenible en torno al desarrollo de la sostenibilidad empresarial como la estrategia de negocios del siglo XXI, por ser el término correcto aceptado por la Real Academia de la Lengua Española. Según el foro la sostenibilidad es como un sinónimo de filantropía o asistencialismo para la supervivencia humana en las mejores condiciones de vida.

La voz "sustainability" del inglés, en el cual se publican la mayoría de los documentos oficiales de la ONU, significa en español sostenible (Sustain / maintain to: sostener; Webster's Dictionary), y no sustentable, por ello los documentos oficiales de la ONU en español hablan de sostenibilidad y no de sustentabilidad (ReyesSánchez, 2012). Se describe al desarrollo: sostenible y sustentable como conceptos diferentes no consensuados ni concientizados en Latinoamérica entre educadores y científicos. Es vital la discusión en el ámbito educativo para de ahí decantar a la ciudadanía el concepto correcto de desarrollo "sustainable" dado por la ONU en 1987. La Tabla 1 define: Crecimiento, desarrollo, sostener, sustentar, sostenible, sustentable, sostenibilidad, sustentabilidad, desarrollo sustentable, desarrollo sostenible y el término inglés "sustainable" (La Tabla 1 fue tomada del artículo de Reyes-Sánchez (2012) con algunas inclusiones adicionales).

Sistemas económicosociales sustentables (supportable system) han de ser reproducibles más allá del corto plazo, sin deteriorar sus propios ecosistemas, proporcionándoles los medios de persistencia de forma exógena: extrayendo la materia y la energía de la que se alimentan para desarrollarse, a costa de otros 
sistemas. El aumento indiscriminado de la entropía negativa del sistema que engulle al sistema proveedor agotándolo, ocasiona una entropía global negativa contraria a la Segunda Ley de la Termodinámica e implica llegar a una situación de crisis. En la Tabla 2 se recopilan los términos ingleses Sustainaible, sustainably y sustainability según el diccionario Webster's Dictionary (Webster's Online Dictionary, 2012).

\section{Tabla 1. Conceptos del crecimiento social debido a la globalización}

\begin{tabular}{|c|c|}
\hline Crecir & $\begin{array}{l}\text { Mide el incremento en la actividad económica en el corto plazo, sin importar la situación social y } \\
\text { ambiental en que viven las personas. El crecimiento se basa en los recursos naturales y sociales de } \\
\text { que dispone, imposible pensar en crecimiento a largo plazo ya que la capacidad de carga de la } \\
\text { Tierra tiene un límite, independientemente de las mejoras tecnológicas que se sobrevengan. } \\
\text { Aumento económico sin desarrollo social y ambiental. }\end{array}$ \\
\hline Desarrollo & $\begin{array}{l}\text { Desarrollo económico a corto y largo plazo que satisface las necesidades del ser humano sin } \\
\text { afectar la carga de los ecosistemas. Necesidades fundamentales de la sociedad: la educación, } \\
\text { necesidades culturales, espirituales etc. y no sólo las económicas. Es un mejoramiento de su } \\
\text { calidad de vida. Aumentar la habilidad de cada uno para construir su propia visión del futuro. } \\
\text { Aumento económico con desarrollo social y ambiental. }\end{array}$ \\
\hline $\begin{array}{l}\text { Sostener* } \\
\text { Del lat. } \\
\text { sustinēre }\end{array}$ & $\begin{array}{l}\text { Verbo. Mantener firme algo, mantenerse en un medio o en un lugar sin caer o haciéndolo } \\
\text { lentamente. } \\
\begin{array}{ll}\text { 1. tr. Sustentar, mantener firme algo } & \text { 2. tr. Sustentar o defender una proposición } \\
\text { 3. tr. Sufrir, tolerar. Sostener los trabajos } & \text { 4. tr. Prestar apoyo, dar aliento o auxilio } \\
\text { 5. tr. Dar a alguien lo necesario para su } & \text { 6. tr. Mantener, proseguir. Sostener } \\
\text { manutención } & \end{array}\end{array}$ \\
\hline $\begin{array}{l}\text { Sustentar* } \\
\text { Del lat. } \\
\text { sustentāre, int. } \\
\text { de sustinēre }\end{array}$ & $\begin{array}{l}\text { Verbo. Proveer a alguien del alimento necesario; conservar algo en su ser o estado } \\
\begin{array}{ll}\text { 1. tr. Conservar algo en su ser o estado. } & \text { 2. tr.Apoyar. } \\
\text { 3. tr. Defender o sostener determinada opinión. } & \text { 4. tr. Sostener algo para que no se caiga o se } \\
& \text { tuerza. }\end{array}\end{array}$ \\
\hline Sos & $\begin{array}{l}\text { Adjetivo. Dicho de un proceso: Que puede mantenerse por sí mismo (sin agotar sus recursos), } \\
\text { como lo hace, p. ej., un desarrollo económico sin ayuda exterior ni merma de los recursos } \\
\text { existentes. (Dicc. Clave). }\end{array}$ \\
\hline Suste & $\begin{array}{l}\text { Adjetivo. Que se puede sustentar o defender con razones (Dicc. de la Real Academia Española } \\
\text { DRAE). } \\
\text { Concepto de orden económico, interpretación economicista del desarrollo que genera desarrollismo. } \\
\text { Se basa en la sustracción de recursos para alimentarse y generar desarrollo en detrimento de los } \\
\text { países, regiones o sociedades de quienes se alimenta; sin importar los costos sociales y } \\
\text { ambientales de los seres y naciones de donde extraen dichos recursos. }\end{array}$ \\
\hline Soste & $\begin{array}{l}\text { Sustantivo femenino. Cualidad de sostenible (DRAE). } \\
\text { Es un principio de carácter antropocéntrico que expresa nuestro respeto por las generaciones } \\
\text { humanas futuras con equidad y justicia; nuestro deseo por preservar, el mayor tiempo posible las } \\
\text { condiciones de vida en la Tierra para todos los seres vivos. } \\
\text { En economía y ecología (Dicc. Clave) capacidad para desarrollarse a lo largo del tiempo sin agotar } \\
\text { los recursos naturales, causando el mínimo impacto medioambiental posible: La sostenibilidad } \\
\text { requiere un equilibrio entre la satisfacción de nuestras necesidades y el mantenimiento de los } \\
\text { recursos existentes, como el agua, la madera o el carbón. }\end{array}$ \\
\hline Sustentabilidad & La palabra sustentabilidad no está en el diccionario. \\
\hline $\begin{array}{l}\text { Desarrollo } \\
\text { sostenible }\end{array}$ & $\begin{array}{l}\text { Antropocéntrico e integral. Satisface las necesidades de la generación presente sin comprometer la } \\
\text { capacidad de las generaciones futuras, para satisfacer sus propias necesidades. La actividad } \\
\text { humana no debe sobrecargar las funciones ambientales, ni deteriorar su calidad. Se reconoce y } \\
\text { respeta la naturaleza integral e interdependiente de la Tierra. }\end{array}$ \\
\hline $\begin{array}{l}\text { Desarrollo } \\
\text { sustentable }\end{array}$ & $\begin{array}{l}\text { Desarrollismo. Confort de los países desarrollados a costa del deterioro de los recursos (materiales, } \\
\text { energéticos y sociales) del tercer mundo. }\end{array}$ \\
\hline Sustainable: & $\begin{array}{l}\text { ponde a una notación dinámica y positiva: To keep going } \\
\text { Avanzar continuamente, resistir, sin ceder. }\end{array}$ \\
\hline
\end{tabular}

Rodríguez y Govea (2006) ven al desarrollo sustentable y sostenible como dos vertientes de desarrollo. La Tabla 3 resume el desplazamiento del desarrollo sustentable al sostenible, considerando: el rol de la 
economía, la concepción sobre la naturaleza después del tratado de Kioto; el papel de la tecnología; las principales políticas para alcanzar el desarrollo y las doctrinas que soportan los enfoques.

Se han establecido: Principios de Sostenibilidad (James y Lathi, 2003; Tuazon et al., 2012) como: Reducir la dependencia de los combustibles fósiles y la explotación de las minas de metales y minerales, reducir la dependencia de materiales, químicos sintéticos y de otras sustancias no naturales, reducir la invasión a la naturaleza y su devastación y satisfacer las necesidades humanas con justicia y eficientemente. Principios de química verde (química sostenible) (Anastas y Eghbali, 2010) como: prevenir generación de residuos, síntesis químicas menos peligrosas, eficiencia energética, materia prima renovable, productos biodegradables entre otros. El 10 de marzo de 2008, el regente del Tribunal de la Penitenciaria Apostoliza del Vaticano, cardenal Gianfranco Girotti, presentó a la iglesia católica una lista de nuevos pecados capitales o sociales (Hernández, 2008), se mencionan algunos: Contaminar el medio ambiente, provocar injusticia social, causar pobreza, enriquecerse hasta límites obscenos a expensas del bien común, entre otros.

Tabla 2. Definición de términos según el diccionario ingles Webster

\begin{tabular}{|l|l|}
\hline Término inglés (español) & Descripción \\
\hline Sustainable (sostenible) & Adjetivo: \\
& $\begin{array}{l}\text { 1: Capaz de ser sostenido } \\
\text { 2: a) De, relacionada con, o ser un método de recolección o uso de un recurso } \\
\text { para que el recurso no se agota ni dañe permanente. } \\
\text { b) De, o relacionado con un estilo de vida que implica el uso de métodos } \\
\text { sostenibles. }\end{array}$ \\
\hline Sustainably (calidad de sostenible) & $\begin{array}{l}\text { Adverbio: } \\
\text { (adverbio: sostenible) }\end{array}$ \\
$\begin{array}{l}\text { 1. En una constante, firme, de manera eterna, permanente o inmutable } \\
\text { 2. Inflexión adverbial de uso poco frecuente del adjetivo sostenible }\end{array}$ \\
\hline Sustainability (sostenibilidad) & $\begin{array}{l}\text { Sustantivo: } \\
\text { 1. La calidad de ser sostenible. }\end{array}$ \\
\hline
\end{tabular}

Tabla 3. Desarrollo Sustentable/Sostenible desde la década de los 60 en adelante

\begin{tabular}{|c|c|c|c|c|c|c|}
\hline Enfoques & $\begin{array}{l}\text { Rol de la } \\
\text { Economía }\end{array}$ & $\begin{array}{l}\text { Concepción de } \\
\text { la Naturaleza }\end{array}$ & $\begin{array}{l}\text { Papel de la } \\
\text { Tecnología }\end{array}$ & Política & Metarrelato & Doctrina \\
\hline Sustentable & $\begin{array}{c}\text { Crecimiento } \\
\text { económico } \\
\text { ilimitado } \\
\text { antinaturalismo. }\end{array}$ & $\begin{array}{l}\text { Explotación de } \\
\text { todos los } \\
\text { recursos, la } \\
\text { tecnología } \\
\text { resolvería la } \\
\text { escasez. }\end{array}$ & $\begin{array}{l}\text { Soluciones } \\
\text { técnicas mixtas } \\
\text { para revertir los } \\
\text { efectos de la } \\
\text { degradación } \\
\text { entrópica. }\end{array}$ & $\begin{array}{c}\text { Ciertas } \\
\text { reestructura- } \\
\text { ciones de las } \\
\text { instituciones. } \\
\text { Modificación mero } \\
\text { proteccionismo } \\
\text { Conservacio- } \\
\text { nismo. }\end{array}$ & $\begin{array}{l}\text { Promover } \\
\text { crecimiento } \\
\text { económico. } \\
\text { Conservacio- } \\
\text { nismo } \\
\text { ambiental. }\end{array}$ & $\begin{array}{l}\text { Antropocén } \\
\text { trica. }\end{array}$ \\
\hline Sostenible & $\begin{array}{c}\text { Desarrollo } \\
\text { económico } \\
\text { Social } \\
\text { ambientalmente } \\
\text { adecuado. } \\
\text { Ambientalismo. } \\
\text { Ecoambiental. } \\
\text { Socioambiental. } \\
\text { Sociocultural } \\
\text { Ambiental. }\end{array}$ & $\begin{array}{c}\text { Gestión y } \\
\text { protección del } \\
\text { medio ambiente y } \\
\text { la biodiversidad. } \\
\text { Reapropiación } \\
\text { sociocultural de la } \\
\text { naturaleza. }\end{array}$ & $\begin{array}{l}\text { Tecnologías } \\
\text { limpias. } \\
\text { Patrones } \\
\text { tecnológicos } \\
\text { que no } \\
\text { deterioren el } \\
\text { medio } \\
\text { Ambiente. } \\
\text { Revalorización } \\
\text { cultural de la } \\
\text { Tecnología. }\end{array}$ & $\begin{array}{l}\text { Democratización } \\
\text { con una gestión } \\
\text { participativa no } \\
\text { solo económica } \\
\text { sino política } \\
\text { cultural, social y } \\
\text { gerencial. } \\
\text { Gobernanza. } \\
\text { Control Social. }\end{array}$ & $\begin{array}{l}\text { Valores Éticos. } \\
\text { Compromiso. } \\
\text { Responsabili- } \\
\text { dad_Social } \\
\text { Compartida. } \\
\text { Empodera- } \\
\text { miento } \\
\text { Ciudadano. }\end{array}$ & $\begin{array}{c}\text { Conciencia } \\
\text { Cívica. } \\
\text { Capacidad } \\
\text { Asociativa. } \\
\text { Confianza. } \\
\text { Conducta } \\
\text { Ética. } \\
\text { Creatividad. } \\
\text { Conocimiento } \\
\text { proactivo. }\end{array}$ \\
\hline
\end{tabular}

\section{RESULTADOS Y DISCUSIÓN}

Naredo (1997) muestra una sostenibilidad débil que corresponde a la economía tradicional del crecimiento económico y la sostenibilidad fuerte que se basa en las economías: física que toma en cuenta la termodinámica de los sistemas y la de la naturaleza o ecológica para que en conjunto mantengan la salud del medio ambiente y del cual depende la vida y la economía humana. Para Norton (1992) la sostenibilidad 
es la realización de las necesidades humanas hoy y asegurar las del mañana y añade la sostenibilidad ecológica como la descrita por Naredo de protección al medio ambiente. De aquí que la sostenibilidad fuerte de Naredo y ecológica de Norton es la que responde al bienestar social, económico y ambiental establecido en la Cumbre de la Tierra "Satisfacción de las necesidades humanas respetando su medio ambiente".

Naredo (1997). Se refiere a la sostenibilidad parcial y a la global como subsistema y sistema respectivamente y que de acuerdo a los conceptos de sostenibilidad anteriores si el subsistema es sostenible lo será el sistema global (que lo contiene) de lo contrario el sistema se volverá insostenible. Las exigencias humanas a vivir con confort y más allá superfluamente por ambición, han deteriorado la calidad de vida de otros que menos tienen y han agotado los sistemas naturales, por lo que más que una moda la sostenibilidad es una exigencia. Y como lo describe Pearce (1989) esta sostenibilidad debe ser sin declive de recursos ni daños ambientales y con estabilidad social. Daly (1994) Define una sociedad sostenible en base a sus recursos renovable y no renovables (dando algunos principios de sostenibilidad) como la que permite la regeneración de sus recursos naturales que usa, cuida que la cantidad de contaminantes emitidos no sean superiores a los limites que la naturaleza permita sin deteriorarse. Define un capital: no renovable y un humano, estableciendo el principio de mesura en el uso del capital natural no renovable para permitir al capital humano creador encuentre su sustitución. La CEPAL (2002) pone en evidencia (como los anteriores autores) de que el progreso económico se base en la cantidad de recursos naturales suficientes, renovables y reemplazables antes de su agotamiento y el medio ambiente sano para alcanzar un desarrollo verdaderamente sostenible. Según la Asociación Americana Para el Desarrollo de la Ciencia, el requerimiento de satisfacer las necesidades humanas y al mismo tiempo preservar los recursos naturales nos lleva a un dilema ya que ambas metas son difíciles de alcanzar al mismo tiempo. Como ejemplo se tiene el modelo capitalista de producción y consumo que se basa en la explotación de recursos naturales.

Rodríguez y Govea (2006) hablan de 3 vertientes del desarrollo sostenible: $1^{\mathrm{a}}$. La sociedad apoyada en sus raíces. $2^{\mathrm{a}}$. Una sociedad pensada por el gobierno en conjunto con la ciudadanía a través de la democracia hacia una visión de logros colectivos. $3^{\mathrm{a}}$. El factor medioambientalista haciendo sostenible la triada sociedad-economía-medio ambiente. Estas últimas vertientes deben basarse en valores éticos y conocimientos que deberán adquirirse para que con conciencia y compromiso se construyan sociedades sostenible que utilicen los recursos adecuadamente y con pertinencia sean menos depredadores, usen procesos limpios, tengan una cultura de gestión política y empresarial comprometida y responsable con leyes sostenibles acordadas con la ciudadanía, para alcanzar la sostenibilidad social, económica y ambiental.

En contraparte al concepto de sostenibilidad surge el término sustentabilidad que aunque no existe en el diccionario de la Lengua Española, es el modelo productivo que no respeta al capital natural, lo explota indiscriminadamente y no es deseado para el futuro humano pero que está pasando. Es motivo de reuniones de grupos gubernamentales en el marco de la ONU (Nuestro futuro común, Agenda 21, Rio+20, etc.) y sociales como el ambientalista Greenpeace, para hacer consiente a la gente del deterioro del planeta y la urgencia de tomar medidas a corto y largo plazo por la vida sostenible. Se conoce también como desarrollo insostenible o soportable. Leff (2003) acepta la diferencia entre desarrollo sostenible y sustentable pero advierte que ambas ocultan la real crisis ambiental y ecológica derivada de la actividad económica, habla en este sentido del modelo capitalista como degradador del ambiente, de una sociedad depredadora que no repara su entorno, es utilitarista en el sentido de su bienestar egoísta sin importar el de los demás, falta del sentido de nosotros.

En México, algunas instituciones educativas resolvieron el debate entre sostenible y sustentable con el análisis lingüista y semántico de los conceptos y eligieron el término sostenible como el que se refiere al establecido para la permanencia de la vida actual y futura en un medioambiente sano. Márquez R. A. (2000) analizando la estructura lingüística o gramatical de las palabras sustentable y sostenible dice que son adjetivos verbales que derivan de los respectivos verbos: sostener y sustentar mediante el agregado a la raíz del verbo del sufijo -able o -ible que denota la idea de capacidad o aptitud para recibir la acción del verbo. Esto quiere decir que sostenible es lo que es capaz de sostener o de sostenerse y sustentable aquello que es capaz de sustentar o sustentarse. Aplicando estas observaciones a la frase desarrollo, resulta que desarrollo sostenible es el desarrollo que puede o es capaz de sostenerse, y desarrollo sustentable es el que puede o es capaz de sustentarse. Gramaticalmente el uso de ambos adjetivos como calificativos del sustantivo desarrollo es válido. Pero habría que definir si semánticamente, es decir, desde el punto de vista del significado, los dos son igualmente válidos, o si sólo uno de ellos puede emplearse con propiedad. Por lo que debe analizarse al significado de los dos verbos. Sostener, dice el DRAE (2012), es sustentar, mantener firme una cosa. Tiene otras acepciones, pero ésta es la que interesa ahora. En cuanto a sustentar, el mismo DRAE lo define en una de sus acepciones como sostener una cosa para que no se caiga o se tuerza. Es así que el diccionario ve a estos verbos como sinónimos casi perfectos: mantener firme una cosa equivale a sostener una cosa para que no se caiga. Se sobreentiende que aquella cosa que 
debe sostenerse o sustentarse existe de antemano, es decir, que el sostenimiento o sustentación de la misma no es lo que le da origen, sino que, una vez presente dicha cosa, se pretende mantenerla, sostenerla, asegurar su continuidad. Se trata de que el desarrollo, una vez iniciado, se mantenga, se conserve, incluso que aumente o se acelere. Desde este punto de vista se concluye que estrictamente en el terreno del lenguaje, del léxico y la semántica, los adjetivos sostenible y sustentable son sinónimos, como lo son los verbos de que derivan, y por tanto su uso es indistinto, sólo sobre la base del gusto del usuario, sin que el empleo de uno u otro obedezca a regla alguna. Sin embargo, interesa establecer, dentro del ámbito científico del concepto (tecnicismo), algún matiz semántico (significado, sentido o interpretación), alguna diferencia conceptual entre lo sostenible y lo sustentable. En el DRAE aparece el adjetivo sustentable (que se puede sustentar o defender con razones), pero no sostenible. En cambio, en Clave (2012), diccionario de uso del español actual, no figura sustentable, pero sí sostenible que en su primera acepción lo define como: que puede ser sostenido y agrega como ejemplos: 1) "El mundo debe tener un desarrollo sostenible, porque el progreso indiscriminado está causando graves desastres ecológicos, 2) en economía y ecología: "desarrollo que puede mantenerse a lo largo del tiempo sin agotar los recursos naturales, causando el mínimo impacto medioambiental posible: Si conseguimos limitar la explotación de los bosques y el consumo de madera, estaremos favoreciendo un desarrollo sostenible". El mismo diccionario Clave señala que el adjetivo sostenible se refiere a: "un proceso que puede mantenerse por sí mismo como p. ej., un desarrollo económico sin ayuda exterior ni merma de los recursos existentes". En la declaración de Rio de Janeiro de 1992, se define el desarrollo sostenible (sustainable) como: "aquel desarrollo que satisface las necesidades de las generaciones presentes sin comprometer las posibilidades de las generaciones futuras para atender sus propias necesidades". Aquí puede verse ese matiz semántico que bien pudiera establecer una cierta diferencia entre lo sostenible y lo sustentable. Coen, A. A. (2006) dice que el truco semántico está en separar, entre las acepciones de los verbos sostenible y sustentable, por una parte las que sólo implican 'asentamiento', 'base', 'apoyo', 'sostén', 'firmeza', 'seguridad', y por la otra parte las que solamente supongan 'alimentación', 'nutrimento', 'manutención'. Así tendremos que sostenible se refiere al aspecto endoestructural del sistema de que se trate, lo que ha de permanecer firmemente establecido, asentado, fijo, inalterable, inamovible. Y sustentable será lo supra - o superestructural de ese mismo sistema, lo que requiere que se lo esté alimentando, proporcionándole los medios de sobrevivencia y de persistencia, a fin de que pueda extender su acción, no sólo en su ámbito (espacio) sino también en el tiempo.

Los documentos de la ONU en español usan el término sostenibilidad. Reyes-Sánchez, (2012) distingue al desarrollo sostenible del sustentable, afirmando que el dado por la ONU como "sustainable development" es el sostenible y debe ser analizado entre los docentes para transmitirlo a la ciudadanía. En Venezuela, Rodríguez y Govea ven al desarrollo: sostenible y sustentable como vertientes distintas: El sostenible es antropocéntrico e integral (con valores, tecnologías limpias y leyes de protección) que proporciona bienestar a las presentes y futuras generaciones sin deterioro ambiental-social-económico. El desarrollo sustentable es también antropocéntrico pero sin atender el deterioro ambiental-social-económico que ocasiona (externalidades) creando brechas entre países desarrollados y subdesarrollados. Lleva a una crisis global.

Sostenibilidad y dimensión social: Para que una sociedad sea sostenible ha de frenar el crecimiento demográfico, mejorar la economía de cada país, erradicar la pobreza fortaleciendo las capacidades de poblaciones vulnerables para generarles oportunidades de crecer por sí mismas y superar la inopia en que viven y finalmente dar educación ambiental. Para lograr un desarrollo sostenible, debe hacerse un buen trabajo en materia de gestión ambiental a nivel internacional y cooperante entre los estados (Strange y Bayley, 2009). Las medidas hacia la sostenibilidad deben ser de tipo preventivo y correctivo. A partir de los conceptos dados por Reyes-Sánchez, y Rodríguez y Govea puede pensarse que un sistema sostenible es autosuficiente en recursos, cerrado termodinámicamente y con entropía de equilibrio $\Delta \mathrm{S}=0 \mathrm{Fig} .1$.

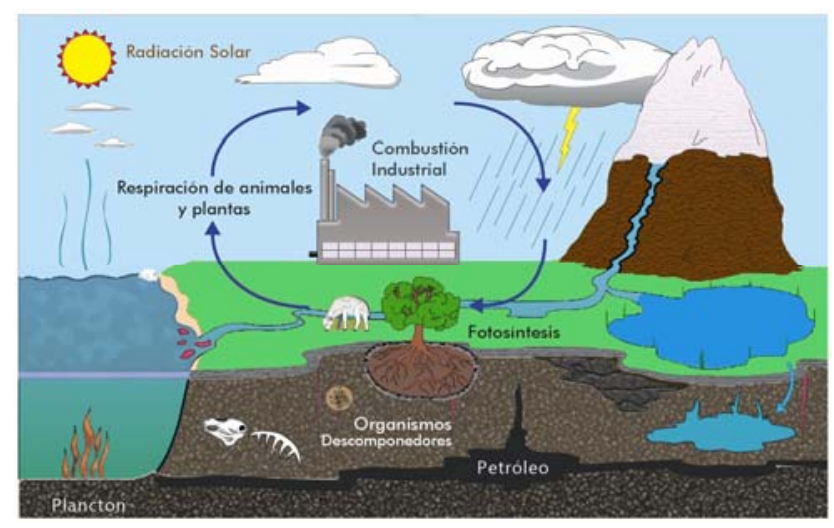

Fig.1: Sistema sostenible, autosuficiente que puede mantenerse en el tiempo sin ayuda exterior. 
Un sistema sustentable es abierto termodinámicamente, necesita se le den los medios para persistir sin deterioro -o con un mínimo menoscabo- a través del tiempo (país desarrollado con entropía negativa $\Delta S<0$ ). Se alimenta de los recursos de otros sistemas (países subdesarrollados con entropía creciente $\Delta S>0$ ). Crece a costa del deterioro de los sistemas que le dan la energía Fig. 2. Los nuevos pecados capitales o sociales surgen del desarrollismo, de la globalización de las sociedades y efectivamente, si se vive en la insostenibilidad y si la basura no se recicla, la humanidad se irá al infierno (no sobrevivirá).

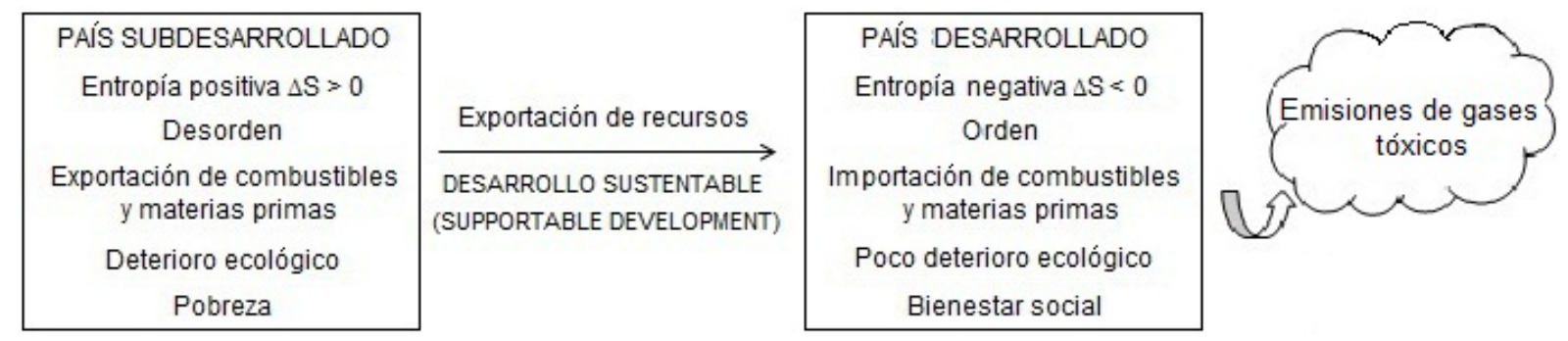

Fig. 2: Sistema sustentable: país desarrollado que se "alimenta" de los recursos de países subdesarrollados.

Resumen del análisis documental. La traducción al español de la voz inglesa sustainable es sostenible. En Latinoamérica se distingue desarrollo sostenible y sustentable. El desarrollo sostenible es antropocéntrico e integral con valores, tecnologías limpias y leyes de protección que proporciona bienestar a las presentes y futuras generaciones sin deterioro ambiental-social-económico. Tiene futuro, una política ecológica ambiental y la necesidad de acelerar el progreso socioeconómico de los países menos desarrollados (ecologización de la economía). La sostenibilidad está compuesta de sistemas y subsistemas, con recursos humanos, naturales y manufacturados que obliga a establecer principios. Los sistemas sostenibles, son sistemas ideales (el paraíso) no alcanzables en países subdesarrollados o en vías de desarrollo.

Se ha dado el término desarrollo sustentable al crecimiento económico que negocia la compra de recursos naturales sobre todo del tercer mundo (mercantilismo ecológico), que no se regeneran o sustituyen. Busca la eficiencia en el uso de los recursos mediante la tecnología, el incremento de bienes y servicios sin tener en cuenta el deterioro ambiental y del bienestar social de las personas que menos tienen y que llevaría a un colapso del sistema. Los países del primer mundo son ejemplo de sistemas sustentables, que mantiene su confort a costa de alimentarse de recursos de países tercermundistas que se deterioran en el tiempo. Es lo más cercano a la realidad. El desarrollo sustentable es un desarrollo insostenible de la sociedad que conduce a nuevos pecados capitales que llevarán a la humanidad a una crisis. Se crean muchos problemas: el uso insuficiente de la energía, la falta de conservación del agua, el aumento de contaminación, los abusos de los derechos humanos, el uso excesivo del transporte personal, el consumismo, etc.

Tomado de la reseña de Fernández, del libro En Cuidar la T(tierra) "Jorge Riechmann matiza y sugiere traducir la voz inglesa sustainable por durable, inspirándose en el uso que hizo de este adjetivo el poeta José Martí (Fernández, 2003)".

\section{CONCLUSIONES}

Del análisis de las reflexiones de los documentos expuestos en este trabajo, se pueden obtener las siguientes conclusiones: 1) el término "sustentable" es un pochismo del inglés "sustainable" que circula ampliamente y ha acabado incorporándose al uso normal, como lo utiliza Bustillo-García y Martínez-Dávila, (2008) o como lo define el diccionario Clave en la acepción 2, "en zonas del español meridional se usa sustentable", 2) el término "sostenible" es el establecido en el informe Brundtland del cuidado y mantenimiento de los recursos naturales que proporcionan bienestar a la humanidad hoy y mañana extendiéndose el concepto a las relaciones humanas sin violencia, equitativas y sin pobreza, 3) la crítica al término sustentable establece que es parecido al capitalismo donde los que menos tienen trabajan para satisfacer las necesidades de los que más tienen en una sociedad no igualitaria, explotada en sus recursos humanos y naturales, 4) esta investigación de términos es importante, los docentes deben aclararlos, para de esta forma contribuir a una mejor educación.

\section{REFERENCIAS}

Acuerdo por un México Sostenible (en línea), (2011): Foro Negocios Verdes. Cd. de México en México. http://www.igs.org.mx/sites/default/files/AcuerdoMexicoSostenible.pdf. Acceso: 22 de diciembre (2012). 
Anastas, P. y Eghbali, N., Green Chemistry: Principles and Practice, Chem. Soc. Rev. (base de datos en línea), 39, 301-312 (2010). http://www.bidi.uam.mx:3096/en/content/articlehtml/2010/CS/B918763B. Acceso: 17 de enero (2013).

Bustillo-García, L. y Martínez-Dávila, J. P., Los enfoques del desarrollo sustentable, Interciencia (base de datos en línea), 33(5), 389-395 (2008).

Castañeda, B., An index of Sustainable Economic Welfare (ISEW) for Chile, Ecological Economics: 28, 231244 (1999). En la base de datos EBSCO: SocIndex with full text. Acceso: 16 de enero (2013).

CEPAL y PNUMA. La sostenibilidad del desarrollo en América Latina y el Caribe: Desafíos y oportunidades. Libros de Cepal, 68, Santiago de Chile, Chile (2002).

Clave. SM diccionarios. Diccionario Clave (en línea). http://portada.smdiccionarios.com/app.php. Acceso: 24 de diciembre (2012).

Coen, A. A., De sostenible y sustentable, Correo del Maestro (en línea), 116 (2006). http:/www.correodelmaestro.com. Acceso 15 de enero (2013).

Cumbre de la Tierra. The United Nations Conference on Environment and Development (en línea), Having met at Rio de Janeiro, Brazil June 3-14 (1992). http://habitat.igc.org/agenda21/rio-dec.htm. Acceso: 14 de enero (2013).

Daly, H., Investigación agraria: Economía: Vols. 9-10. Instituto Nacional de Investigaciones Agrarias. (1994).

DRAE, Diccionario de la lengua española, 22 ${ }^{\text {ra }}$ edición, (en línea). Espasa-Calpe, Madrid, España (2012). www.rae.es/drae. Acceso: 22 de diciembre (2012).

Educación para la sostenibilidad. Década por una educación para la sostenibilidad (en línea) (2005). http://www.oei.es/decada/compromiso.php. Acceso: 15 de enero (2013).

Falconi F., Economía y Desarrollo Sostenible. ¿Matrimonio feliz o divorcio anunciado? El caso de Ecuador, pp 213. FLACSO, Quito, Ecuador. (2002).

Fernández, B, F., « Jorge Reichmann, Cuidar la T(i)erra. Políticas agrarias y alimentarias sostenibles para entrar al siglo XXI, Icaria, Barcelona, pp 623 (2003). », Polis (en línea), 10 | 2005, Puesto en línea el 12 noviembre 2012. http://polis.revues.org/7617. Acceso: 14 de enero (2013).

Garmendia, E., Prellezo, R., Murillas, A., Escapa, M., y Gallastegui, M., Weak and strong sustainability assessment in fisheries. Ecological Economics (en línea): http://dx.doi.org/10.1016/j.ecolecon.2010.08.001. 70(1), 96-106 (2010).

Hernández, V. I., Quien no recicle basura ira al infierno (en línea), (2008). http://www.elmundo.es/elmundo/2008/03/11/ internacional/1205200007.html. Acceso: 22 de diciembre (2012).

James, S. y Lathi, T., Eco-municipalities: Sweden and the United States: A Systems Approach to Creating Communities (en línea), (2003). http://www.knowledgetemplates.com/sja/ecomunic.htm. Acceso: 22 de diciembre (2012).

Kates, R. W. y otros veintidós autores, Policy fórum: Environmental and development-Sustainability Science, Science (en línea): 292 (5517), 641-642 (2001).

Leff, E. G., La geopolítica de la biodiversidad y el desarrollo sustentable: Economización del mundo, racionalidad ambiental y reapropiación social de la naturaleza. Revista Líder: 11, 21-37. Osorno: Universidad de los Lagos (2003).

Márquez, R. A., Sostenible y sustentable, Analítica. com (en línea), (1200). http://www.analitica.com/bitblio/amarquez/sostenible.asp. Acceso: 12 de enero (2013).

Naredo, J. M., Sobre el origen, el uso y el contenido del término sostenible, Ciudades para un Futuro más Sostenibles, (en línea), (1997). http://habitat.aq.upm.es/cs/p2/a004.html. Acceso: 22 de diciembre (2012). 
Norton, B. B., Sustainability, Human Welfare and Ecosystem Health (en línea), Environmental Values: 1(2), 97-111 (1992).

Novo, M., La educación ambiental: una genuina educación para el desarrollo sostenible. Revista de educación, No Extraordinario 1, 195-217 (2009). Dialnet-Universidad de la Rioja http://www.revistaeducacion.mec.es/re2009/re2009_09.pdf. Acceso: 14 de enero (2013).

ONU. Nuestro futuro común: Informe Brundtland (en línea), 1987. http://www.un-documents.net/wcedocf.htm. Acceso: 14 de enero (2013).

Pearce, D. W., Markandya, A. y Barbier, E, Blueprint for a Green Economy, Earthscan Publications, London, U. K (1989).

Redclift, M., Sustainable Development Exploring the Contradictions. Routledge. Londres, RU. 222 pp. (1987).

Reyes-Sánchez, L. B., APORTACIÓN DE LA QUÍMICA VERDE A LA CONSTRUCCIÓN DE UNA CIENCIA SOCIALMENTE RESPONSABLE, Educ. quím.: 23(2), 222-229 (2012).

Rodríguez, I. y Govea, H., El discurso del desarrollo sustentable en América Latina, Revista Venezolana de Economía y Ciencias Sociales (en línea), 12(2), (2006).

Strange, T. y Bayley, A., What is Sustainable Development? In Sustainable Development: Linking Economy, Society, Environment, OECD Publishing (2009). http://dx.doi.org/10.1787/9789264055742-3-en. Acceso 17 de enero (2013).

Tuazon, D., Corder, G., Powell M. y Ziemski M., A practical and rigorous approach for the integration of sustainability principles into the decision-making processes at minerals processing operations, Minerals Engineering: 29, 65-71 (2012).

UNESCO, International Congress on Environmental Education and Training (en línea), UNESCO-UNEP Environmental Education Newsletter Vol. XII, No 3, Moscú/París (1987).

Webster's Dictionary, Webster's Online Dictionary, 2012. http://www.websters-online-dictionary.org/. Acceso: 22 de diciembre (2012). 